\title{
Our current approach to root cause analysis: is it contributing to our failure to improve patient safety?
}

\author{
Kathryn M Kellogg, ${ }^{1}$ Zach Hettinger, ${ }^{1}$ Manish Shah, ${ }^{2}$ Robert L Wears, ${ }^{3}$ \\ Craig R Sellers, ${ }^{4}$ Melissa Squires, ${ }^{5}$ Rollin J Fairbanks ${ }^{1}$
}

- Additional material is published online only. To view please visit the journal online (http://dx.doi.org/10.1136/ bmjqs-2016-005991).

For numbered affiliations see end of article.

\section{Correspondence to}

Dr Kathryn M Kellogg, MedStar Health, MedStar Institute for Innovation, Washington, DC 20008, USA; kate.kellogg@ medicalhfe.org

Received 10 August 2016 Revised 29 September 2016 Accepted 21 October 2016 Published Online First 2 February 2017

\section{SLinked}

- http://dx.doi.org/10.1136/ bmjas-2016-005511

- http://dx.doi.org/10.1136/ bmjqs-2016-006229

CrossMark

To cite: Kellogg KM, Hettinger Z, Shah M, et al. BMJ Qual Saf 2017;26:381-387.

\begin{abstract}
Background Despite over a decade of efforts to reduce the adverse event rate in healthcare, the rate has remained relatively unchanged. Root cause analysis (RCA) is a process used by hospitals in an attempt to reduce adverse event rates; however, the outputs of this process have not been well studied in healthcare. This study aimed to examine the types of solutions proposed in RCAs over an 8-year period at a major academic medical institution.
\end{abstract}

Methods All state-reportable adverse events were gathered, and those for which an RCA was performed were analysed. A consensus rating process was used to determine a severity rating for each case. A qualitative approach was used to categorise the types of solutions proposed by the RCA team in each case and descriptive statistics were calculated.

Results 302 RCAs were reviewed. The most common event types involved a procedure complication, followed by cardiopulmonary arrest, neurological deficit and retained foreign body. In 106 RCAs, solutions were proposed. A large proportion (38.7\%) of RCAs with solutions proposed involved a patient death. Of the 731 proposed solutions, the most common solution types were training (20\%), process change (19.6\%) and policy reinforcement (15.2\%). We found that multiple event types were repeated in the study period, despite repeated RCAs.

Conclusions This study found that the most commonly proposed solutions were weaker actions, which were less likely to decrease event recurrence. These findings support recent attempts to improve the RCA process and to develop guidance for the creation of effective and sustainable solutions to be used by RCA teams.

\section{INTRODUCTION}

The problem of morbidity and mortality from adverse events in healthcare has undergone over 15 years of intense scrutiny, funding, regulation and research worldwide. Despite dramatically intensified efforts to increase the safety of the healthcare system, reports have suggested that safety has not improved. The adverse event rate has remained essentially the same, suggesting that our current solutions to the problem are not working. ${ }^{1-10}$ This lack of progress persists despite the devotion of a tremendous amount of financial and human resources at the local, state and national levels in an effort to reduce errors and patient harm. ${ }^{11}$

One common, resource-intensive, practice is the root cause analysis (RCA) process, which is used by most hospitals in the USA. ${ }^{12-15}$ The RCA process has been mandated in response to sentinel events by the Joint Commission since 1997. ${ }^{16}$ Although the RCA process has been presumed to induce change, its effectiveness has been questioned and there is not robust literature to support its efficacy. ${ }^{17}{ }^{18}$ In healthcare, there are reports of difficulty in both determining the causes (more accurately termed the contributing factors) of events and developing and implementing the appropriate corrective actions. ${ }^{13} 19$ The goal of the RCA is twofold. First, the process aims to determine the contributing factors, with a focus on the latent hazards in the system, which contributed to the occurrence of the event. The second is to develop the solutions or proposed changes that, once implemented, will eliminate or reduce the hazard and therefore reduce the chance that a similar event could occur in the future. Previous publications on RCAs have largely involved case reports or editorials, and there have been small studies characterising RCA solutions within a single 
department. ${ }^{20}$ However, few analyses of the process itself are available in the literature, and those that have been done have looked at the types of cases for which RCAs have been performed or the root causes identified by the teams. ${ }^{13} 2122$ Our team has previously examined solution types in a small sample of RCAs, and this study will further contribute by examining event types and solution types in a larger data set. $^{23}$

Although RCAs have been proposed as a mechanism for change, safety scientists believe that the lack of improvement of adverse event rates in healthcare is largely because our methods of approaching change are ineffective. ${ }^{24} 25$ RCAs attempt to offer solutions to effect change, yet few studies have attempted to categorise or evaluate the recommendations that result from the process. ${ }^{25}$ One study showed that fewer than half of RCAs reviewed included recommendations directed at robust system-level improvements. ${ }^{21}$ Many authors have asserted that recommended actions that involve education or policy change are inherently weaker than those that involve redesign of a product or process, as the former are less likely to introduce effective and sustained change into the system. ${ }^{17}$ 26-28 Despite the intent to use RCAs to evaluate systemlevel problems, studies have shown that RCAs in healthcare often focus on attempts to fix individuals rather than on system improvements, while safety engineering fields show us that system-level interventions are more effective. ${ }^{27-29}$

Most states, including New York, require RCAs to be completed after a sentinel event. ${ }^{30}$ Despite the large prevalence of the RCA process in adverse event review and work to identify improved RCA techniques, a systems-based approach to RCAs, and in how to properly respond to the identified causes in a way that will increase system safety in a sustained manner, is not widespread. As a step towards this goal, this study aims to build on prior work by examining the types of solutions proposed in response to RCAs conducted at a large, tertiary care academic medical centre over an 8-year period.

\section{METHODS}

This is a qualitative and quantitative analysis of the content of RCAs performed at one large academic medical centre over an 8-year period. The goal of the study was to determine a taxonomy of solution types proposed by the RCA teams and to determine the number of solution types for all RCAs reviewed. Hospital leadership consented to data review and IRB approval was obtained prior to initiation of data collection.

\section{Setting}

The study was conducted at a 750-bed tertiary care academic medical centre which, at the time of data collection, employed 597 graduate medical trainees across 67 training programmes, and treated 38000 inpatients, 950000 outpatients, 98000 emergency patients and performed 35000 surgeries annually. Mandatory reporting was delivered to a central agency (New York Patient Occurrence Reporting and Tracking System, or NYPORTS) for any occurrence, defined as 'an unintended adverse and undesirable development in an individual patient's condition occurring in a hospital'. ${ }^{30}$

\section{Protocol}

Occurrences were coded by type prior to submission, according to NYPORTS standards, and any occurrence coded as a serious reportable event' required an RCA be conducted. ${ }^{31}$ RCAs were done within 30-60 days following the occurrence. At the study facility, the RCA was led by a member of the quality team from within the office of Quality and Safety. The typical RCA team was six to eight individuals and included one quality officer and at least one member of physician and nursing leadership from each involved or affected discipline. One to two face-to-face meetings were conducted in which involved parties were typically interviewed, with follow-up emails and phone calls used to reach consensus on the final report document. The report was then shared with the hospital's Safety and Risk Management Committee, and the Board of Directors' Quality and Patient Safety Committee and uploaded to NYPORTS, which allows analysis of multi-institutional data. ${ }^{22}$ The RCA team gave a final designation to each case, chosen from one of the following: 'standard of care met, no action needed', 'standard of care met with room for improvement', 'standard of care not met, attributable to systems' or 'standard of care not met, attributable to individual practitioner'.

Full records of all state-reported incidents at the study institution between 2001 and 2008 were obtained from the NYPORTS database in the form of a Microsoft Access database file (Redmond, Washington, USA). Data were deidentified, and a check for duplicates was performed. This database included all information reported by the hospital to the reporting agency during the study period. Data included patient demographic information, a description of the event and the proposed solutions. Only unique reports that reviewed reportable incidents for which an RCA was required were included. Cases designated as 'standard of care met, no action needed', for which no solutions were proposed, were reviewed in the initial analysis for case type but were excluded from the analysis of solution types, and no further analysis of these cases was conducted.

\section{Data analysis}

Once the RCA data were compiled, a severity rating process was performed. In this step, two physicians with safety experience (RJF and MNS) independently 
reviewed details from each case including the study ID number, patient age and gender and the event summary. Each reviewer assigned a severity category to the case outcome, using the previously reported National Coordinating Council for Medication Error Reporting and Prevention error taxonomy (see online supplementary appendix 1), in which errors are rated from A (circumstances or events that have the capacity to become a hazard) to I (patient death). ${ }^{32}$

Raters did not attempt to determine causation but rated the severity of case outcomes based on the information available in the RCA. For cases where ratings were inconsistent by more than two categories, consensus was reached by discussion during a second meeting. If case ratings were off by only one category, the more severe outcome was selected. Subsequently, the data were analysed using Stata (Stata 1C 11, College Station Texas, USA).

Next, a qualitative analysis was performed on the RCA documents. Qualitative research is the optimal method to develop an understanding of an otherwise relatively unknown topic and to capture elements that might be missed in a standardised quantitative approach with previously determined categories. ${ }^{33}$ The complete text from each RCA was imported into Atlas.ti (Berlin, Germany) and reviewed by the qualitative analysis team. All three members of the team (KK, MS, RJF) performed the review for the first 50 cases to generate consistent understandings of the coding definitions. Two members (KK and MS) independently reviewed subsequent cases, and inconsistent results were reviewed by the third member (RJF). Thematic analysis was performed using wellestablished qualitative research methods. ${ }^{34}$ Categories were created and expanded to iteratively develop a codebook. Through this standard process, each event summary was assigned a category. A similar process was undertaken with each RCA solution until each was assigned a solution type. ${ }^{34}$ As part of the same

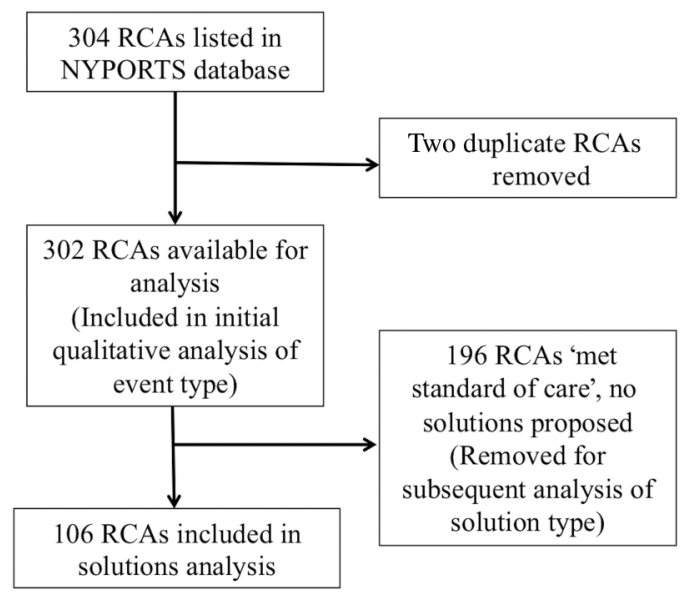

Figure 1 Determination of root cause analyses included in the study sample. project, but subsequent to this analysis, solution-type category definitions for this study have been previously reported by our team. ${ }^{23}$

\section{RESULTS}

Three hundred and two RCA cases were conducted during the 8-year study period. Duplicate records were excluded. All RCAs were included in the initial analysis; subsequently, those cases proposed solutions were included in the solutions analysis (figure 1). Table 1 shows descriptive information for all 302 cases, as well as severity outcome categories for RCAs in which solutions were proposed $(n=106)$. A large proportion (38.7\%) of RCAs with solutions proposed involved a patient death. Adverse events resulting in RCAs occurred throughout the hospital, most frequently in surgical departments (52.6\%), with departments such as psychiatry and neurology submitting cases with the lowest frequency $(2 \%$ and $1.3 \%$, respectively).

The most common event types were procedure complication, cardiopulmonary arrest, neurological deficit and retained foreign body (table 2). The least frequent cases involved electrolyte imbalances, equipment failures or conducting the incorrect study for a patient. During the study period, several event types

Table 1 Characteristics of RCAs performed between 2001 and $2008(n=302)$

\begin{tabular}{|c|c|}
\hline Patient age (mean, SD) & $54(32-66)$ \\
\hline Male (\%) & 49.3 \\
\hline \multicolumn{2}{|l|}{ Hospital departments involved (\%) } \\
\hline Surgery/surgical subspecialties & 52.6 \\
\hline Medicine/medical subspecialties & 13.9 \\
\hline OB/GYN & 7.6 \\
\hline Radiology & 6.3 \\
\hline Paediatrics & 4.3 \\
\hline Anaesthesia & 4.3 \\
\hline Emergency medicine & 4.0 \\
\hline Psychiatry & 2.0 \\
\hline Other & 1.7 \\
\hline Neurology & 1.3 \\
\hline Dentistry & 1.0 \\
\hline \multicolumn{2}{|l|}{ Outcome severity $(n, \%)^{*}$} \\
\hline$A$ & 0 \\
\hline B & 0 \\
\hline C & $8(7.5)$ \\
\hline $\mathrm{D}$ & $17(16.0)$ \\
\hline$E$ & $15(14.2)$ \\
\hline $\mathrm{F}$ & $6(5.7)$ \\
\hline G & $13(12.3)$ \\
\hline $\mathrm{H}$ & $6(5.7)$ \\
\hline 1 & $41(38.7)$ \\
\hline
\end{tabular}


Table 2 Event types for RCAs reviewed between 2001 and 2008

\begin{tabular}{|c|c|c|}
\hline Type & Frequency & Per cent \\
\hline Procedure complication & 63 & 20.7 \\
\hline Cardiopulmonary arrest & 41 & 13.5 \\
\hline Neurological deficit & 35 & 11.5 \\
\hline Retained foreign body & 25 & 8.2 \\
\hline Pulmonary/arterial embolus & 21 & 6.9 \\
\hline Birth complication & 15 & 4.9 \\
\hline Medication administration error & 13 & 4.3 \\
\hline Incorrect procedure/study & 12 & 3.9 \\
\hline Sepsis & 11 & 3.6 \\
\hline Wrong-site surgery/procedure & 9 & 3.0 \\
\hline Devastating illness & 9 & 3.0 \\
\hline Myocardial infarction & 8 & 2.6 \\
\hline Haemorrhage/haematoma & 7 & 2.3 \\
\hline Arrhythmia & 6 & 2.0 \\
\hline Unknown cause of death & 6 & 2.0 \\
\hline Adverse medication event & 4 & 1.3 \\
\hline Compartment syndrome & 3 & 1.0 \\
\hline Fall, inpatient & 3 & 1.0 \\
\hline Event proximate to discharge & 2 & 0.7 \\
\hline Self-harm & 2 & 0.7 \\
\hline Electrolyte disturbance & 2 & 0.7 \\
\hline Assault, inpatient & 1 & 0.3 \\
\hline Bowel perforation & 1 & 0.3 \\
\hline Equipment failure & 1 & 0.3 \\
\hline Sleep apnoea & 1 & 0.3 \\
\hline Ventilator complication & 1 & 0.3 \\
\hline Total & 302 & \\
\hline
\end{tabular}

RCA, root cause analysis.

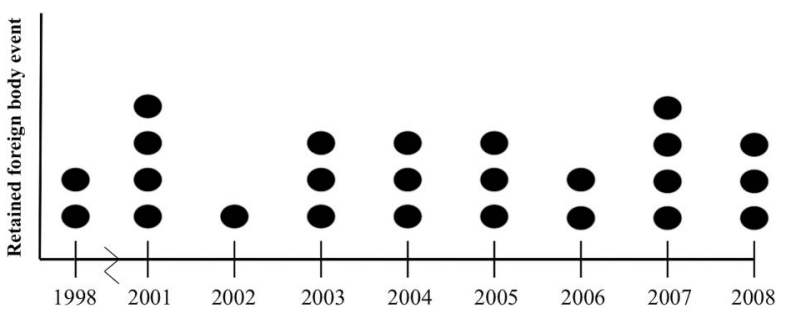

Figure 2 Occurrence of retained foreign body events, as extracted from root cause analyses performed between 2001 and 2008.

were repeated multiple times, including retained foreign body, medication administration error and wrong-site surgery. Figure 2 shows the recurrence of retained foreign body events during the study period, despite RCAs being performed for each of these events.

The average number of solutions proposed per RCA was 4.7, with 499 total solutions identified in the analysis (table 3).

The most common category of solutions was training, most often proposed as didactic teaching,
Table 3 Types of solutions proposed in RCAs between 2001 and 2008

\begin{tabular}{lcc}
\hline Solution type & Frequency $(\mathrm{n}=499)$ & Per cent \\
\hline Training & 100 & 20.0 \\
Process change & 98 & 19.6 \\
Policy reinforcement & 76 & 15.2 \\
Policy change & 44 & 8.8 \\
Counselling & 34 & 6.8 \\
Forms and paperwork change & 28 & 5.6 \\
Physical environment change & 24 & 4.8 \\
IT structure change & 23 & 4.6 \\
Review & 23 & 4.6 \\
Vague & 17 & 3.4 \\
Compliance check/chart review & 13 & 2.6 \\
Institutional change & 9 & 1.8 \\
Contact third party & 5 & 1.0 \\
Risk management & 5 & 1.0 \\
\hline RCA, root cause analysis; IT, information technology.
\end{tabular}

discussions at faculty/staff meetings or via in-service training. For example, in a case where a patient suffered neurological complications from an air embolism that occurred during an interventional radiology procedure, the RCA team stated that, 'The treatment of air embolism should be reviewed annually for the staff in the angio[graphy laboratory] and interventional radiology departments and included as part of the formal teaching of Interventional Radiology residents'. Following a case of intraoperative nerve compression resulting in peroneal nerve injury, one of the proposed solutions was, 'For educative purposes and to heighten awareness of the possibility this could occur due to positioning, this case will be reviewed at Anesthesia's...QI [Quality Improvement] Grand Rounds, and at Orthopedics' QA [Quality Assurance] meeting'.

The second most common solution type proposed was a process change, which could involve a change in workflow, a clinical protocol or procedures around communication. These solutions could involve training staff in the new process. For example, following a case where a patient had a respiratory arrest upon transport to MRI from the Surgical Intensive Care Unit (SICU), a clinical protocol in the form of a written algorithm was developed to determine the stability of SICU patients for transfer for non-urgent diagnostic testing. In another case, an incorrect surgical count prompted the performance of an abdominal $\mathrm{X}$-ray; however, the entire abdomen was not imaged, and thus, a sponge was not identified at the time the $\mathrm{X}$-ray was read. The proposed process change to facilitate communication was, 'The surgeon and the radiologist should collaborate with regard to the plan for imaging the area under review'.

The third most commonly proposed solution was policy reinforcement. In this category, the RCA team 
stated that a policy already in place would be emphasised to staff. For example, in another surgical case of a retained sponge, the RCA team stated, 'Analysis confirmed that our counting policy is effective as written despite this occurrence. However, sponge was retained. Human error determined to be a factor', and thus proposed the following solution: 'Re-emphasise policy and procedures at OR staff meeting and at the next Perioperative Service Chiefs meeting'. In a case where two patients on a unit had the same last name and the wrong patient received a ventilation/perfusion scan, the RCA team proposed the following solution: 'Staff were reminded of the correct procedure for identifying patients'.

\section{DISCUSSION}

This study examined RCAs performed at a major US academic medical centre over an 8-year period. We determined the categories of cases being reviewed by the RCA team, as well as the types of solutions proposed to reduce similar future occurrences. Analyses such as this provide a crucial step to understanding the RCA process and to provide a framework to facilitate future studies that examine the utility of the process. The safety industry recognises that a hierarchy of safety interventions exists, the power of which can lead to greater or lesser improvements in safety. In its most general form, the hierarchy is as follows (from most effective to least); (1) Design changes to remove the hazard, (2) Guard (physically) against the hazard, (3) Warn the personnel about the hazard. ${ }^{35}$ Relatively few of the solutions offered by the RCA teams included design of changes to remove hazards, and we believe that this is representative of RCAs as performed throughout the USA.

We found that in this time period, RCAs were performed for cases from most services in the hospital with the most common being surgical cases. This coincided with the fact that the greatest proportion of cases was related to a procedure complication, the risk for which is higher during surgery than in other hospital settings, as a higher frequency of procedures are performed there. The frequency of different types of cases may also be related to the types of events that are required to be reported in New York. Our data showed a high $(38.7 \%)$ rate of patient death related to the adverse event; however, there is likely under reporting of patients who are less severely injured, making this likely to be an over-representation of overall severity. ${ }^{36}$ In figure 2 , we show that, despite repeated RCA team examination of retained foreign body events, occurrences of that same event continued throughout the study period. While recognising that some types of events are impossible to eliminate completely, we propose that repeat events occur despite repeat RCAs because of the quality and types of solutions that are proposed by RCA teams.
The effectiveness of the RCA process has been questioned in the literature previously, but few studies have critically examined the process as it is used in healthcare to review sentinel events. ${ }^{14}$ Many times, the RCA does not identify meaningful aspects of the event but simply observes that humans are imperfect. For example, failures involving people forgetting something previously known or taught to them simply observes that human memory is imperfect. This finding is trivial and will not contribute to sustainable change without some kind of change in the work setting to support the cognitive work of the healthcare worker or reduce the burden of having to remember critical pieces of information. Additionally, in healthcare, many errors are 'slips' or 'lapses' that occur when the practitioner is in automaticity mode and is not consciously thinking about his or her actions. To begin to address errors due to 'slips' and 'lapses', the conditions under which these errors occur must be identified. ${ }^{37} 38$ Systems-based changes have been found to be most effective in mitigating safety in other industries; however, this has not yet become a standard of practice in patient safety in healthcare.

Our data around policy reinforcement highlights one of the most compelling demonstrations of the ineffectiveness of RCAs, as they have historically been performed in the USA. In what resilience engineers would refer to as the 'work as imagined' space, we see solutions such as reminding staff of the correct procedure, and human error was determined to be a factor. This violates the basic premise of safety engineering involving sociotechnical systems, which recognises that human errors will always be repeated. Just as our parents taught us when we were toddlers, human error is inevitable, thereby proposing a solution for safety mitigation that focuses on reminding people not to make mistakes is an indictment of our approach to safety.

Work done by our team examined the same categories of RCA solutions discussed here and, through front-line personnel and discussions with safety science experts, developed a model of sustainability and effectiveness for solutions. ${ }^{23}$ In that study, the categories developed in this project were used to determine the effectiveness and sustainability of different solutions proposed by RCA teams. This work showed that solutions, such as technology changes and institutional-level changes, were most effective and sustainable, and solutions, such as counselling and disciplinary changes, were the least effective and sustainable. This study can serve as a basis for future analyses of the effectiveness of the RCA process for preventing similar future occurrences.

It is clear that RCA teams need validated tools to use when proposing solutions in order to meet the goal of reducing future similar outcomes. However, no trials have examined the effectiveness of the process or its proposed solutions. ${ }^{10}$ Therefore, more work needs to be done to specifically review 
interventions and evaluate their quality. RCA teams need guidance on solution types and the timing of their implication, and this needs to be validated in order to propose the most effective solutions for change. Work needs to be done to determine how to study near misses and hazards to include information from these events in proposed solutions, as these events are often incredibly informative and predictive of future events but are often not evaluated.

There are limitations of this study, which should be considered when interpreting our results. This study was performed at a single institution. However, this institution performs RCAs in a manner similar to other major medical centres across the country and is in accordance with Joint Commission recommendations. ${ }^{39}$ A multicentre study of RCAs is needed in the future, as studies of the RCA process are scarce. While an 8 -year period is likely representative of the types of RCAs and the solutions proposed, examining a greater length of time might also reveal more about the process. We were unable to review each case in detail and each solution in context, as this information was not submitted to NYPORTS, which may have yielded more information about the derivation of the different solutions.

\section{CONCLUSION}

Our study evaluates the RCA process as performed at a major medical centre, building on prior work to classify and understand the recommendations being made by RCA teams. Our results show that certain event types are seen repeatedly, even following recommendations from RCA teams. Our qualitative analysis reveals that solution types most commonly proposed are not usually the types shown to be more effective and sustainable in other industries. Our study highlights the need for further critical evaluation of the RCA process in healthcare in order to understand if and how the process can be improved to meet the goal of reducing error and increasing patient safety.

\footnotetext{
Author affiliations

${ }^{1}$ MedStar Health, MedStar Institute for Innovation, Washington District of Columbia, USA

${ }^{2}$ BerbeeWalsh Department of Emergency Medicine, University of Wisconsin School of Medicine \& Public Health, Madison, Wisconsin, USA

${ }^{3}$ Department of Emergency Medicine/CSRU, University of Florida/Imperial College London, Jacksonville, Florida, USA

${ }^{4}$ University of Rochester School of Nursing, Rochester, New York, USA

${ }^{5}$ Nationwide Children's Hospital, Columbus, Ohio, USA
}

Correction notice This article has been corrected since it first published Online First. The abstract has been restructured to include a conclusions section.

Acknowledgements Joseph Duckett, Diane Cockrell, Robert Panzer, MD.

Funding This study was funded by the 'Year-Out Research Fellowship' grant from the University of Rochester School of Medicine and Dentistry and by the Emergency Medicine Patient Safety Foundation. At the time of this study, RF was supported by a Career Development Award from the NIBIB, K08EB009090.

Competing interests None declared.

Ethics approval University of Rochester School of Medicine and Dentistry.

Provenance and peer review Not commissioned; externally peer reviewed.

\section{REFERENCES}

1 Wachter RM. Patient safety at ten: unmistakable progress, troubling gaps. Health Aff 2010;29:165-73.

2 Shekelle PG, Pronovost PJ, Wachter RM, et al. Advancing the science of patient safety. Ann Intern Med 2011;154:693-6.

3 Landrigan CP, Parry GJ, Bones CB, et al. Temporal trends in rates of patient harm resulting from medical care. $N$ Engl J Med 2010;363:2124-34.

4 Leape LL, Berwick DM. Five years after To Err Is Human: what have we learned? JAMA 2005;293:2384-90. http://www.ncbi. nlm.nih.gov/entrez/query.fcgi? $\mathrm{cmd}=$ Retrieve $\& \mathrm{db}=\mathrm{PubMed} \&$ dopt $=$ Citation\&list_uids $=15900009$.

5 Wachter RM. The end of the beginning: patient safety five years after “To Err Is Human”. Health Aff 2004;Suppl Web Exclusives: W4-534-545.

6 Brennan TA, Gawande A, Thomas E, et al. Accidental deaths, saved lives, and improved quality. $N$ Engl J Med 2005;353:1405-9.

7 Longo DR, Hewett JE, Ge B, et al. The long road to patient safety: a status report on patient safety systems. JAMA 2005;294:2858-65.

8 Shojania KG, Thomas EJ. Trends in adverse events over time: why are we not improving? BMJ Qual Saf 2013;22:273-7.

9 Baines R, Langelaan M, de Bruijne M, et al. How effective are patient safety initiatives? A retrospective patient record review study of changes to patient safety over time. BMJ Qual Saf 2015;24:561-71.

10 Austin JM, Andrea GD, Birkmeyer JD, et al. Safety in numbers: the development of leapfrog 's composite patient safety score for U. S. Hospitals. J Patient Saf 2013;9:1-9.

11 Provonost P, Ravitz A, Stoll R, et al. Transforming Patient Safety: A Sector-Wide Systems Approach. Report of the WISH Patient Safety Forum 2015. 2015. http://cdn.wish.org.qa/app/ media/1430

12 Williams PM. Techniques for root cause analysis. Proc (Bayl Univ Med Cent) 2001;14:154-7. http://www.ncbi.nlm.nih.gov/ pubmed/16369607.

13 Percarpio KB, Watts BV, Weeks WB. The effectiveness of root cause analysis: what does the literature tell us? Jt Comm J Qual Patient Saf 2008;34:391-8. http://www.ncbi.nlm.nih.gov/ entrez/query.fcgi? $\mathrm{cmd}=$ Retrieve $\& \mathrm{db}=$ PubMed $\& \mathrm{dopt}=$ Citation\&list_uids $=18677870$

14 Commission TJ. Sentinel Event Policy and Procedures. http:/ www.jointcommission.org/Sentinel_Event_Policy_and_ Procedures/

15 Flink E, Chevalier CL, Ruperto A, et al. Lessons learned from the evolution of mandatory adverse event reporting systems. Adv Patient Saf From Res to Implement 2005;3:135-52. http:/ www.ncbi.nlm.nih.gov/books/NBK20547/

16 AHRQ. Root Cause Analysis. Patient Safety Primer. 2014. https:/psnet.ahrq.gov/primers/primer/10/root-cause-analysis

17 Wu AW, Lipshutz AK, Pronovost PJ. Effectiveness and efficiency of root cause analysis in medicine. JAMA 2008;299:685-7. 
18 Polancich S, Roussel L, Patrician P. Best practices for conducting an RCA: are there any? Patient Saf Qual Healthcare 2014. http://psqh.com/september-october-2014/best-practicesfor-conducting-an-rca

19 Peerally MF, Carr S, Waring J, et al. The problem with root cause analysis. BMJ Qual Saf 2016;doi:10.1136/bmjqs-2016-005511.

20 Perotti V, Sheridan MM. Root cause analysis of critical events in neurosurgery, New South Wales. ANZ J Surg 2015;85:626-30.

21 Mills PD, Neily J, Luan D, et al. Actions and implementation strategies to reduce suicidal events in the Veterans Health Administration. Jt Comm J Qual Patient Saf 2006;32:130-41. http://www.ncbi.nlm.nih.gov/entrez/query.fcgi? cmd=Retrieve $\& \mathrm{db}=$ PubMed\&dopt $=$ Citation\&list_uids $=16617944$

22 Faltz LL, Morley JN, Flink E, The New York model: root cause analysis driving patient safety initiative to ensure correct surgical and invasive procedures. In: Henriksen K, Keyes MA, Grady ML, et al, eds. Advances in patient safety: new directions and alternative approaches. Rockville, MD: Agency for Healthcare Research and Quality, 2008.

23 Hettinger AZ, Fairbanks RJ, Hegde S, et al. An evidence-based toolkit for the development of effective and sustainable root cause analysis system safety solutions. J Healthc Risk Manag 2013;33:11-20.

24 Nemeth Cook RC. Reliability versus resilience: what does healthcare need? Hum Factors Ergon Soc Annu Meet Proc 2007;51:621-5. http://www.ingentaconnect.com/content/hfes/ hfproc/2007/00000051/00000011/art00004

25 Choksi VR, Marn C, Piotrowski MM, et al. Illustrating the root-cause-analysis process: creation of a safety net with a semiautomated process for the notification of critical findings in diagnostic imaging.[see comment]. J Am Coll Radiol $2005 ; 2: 768-76$.

26 Wears RL, Fairbanks RJ. Design trumps training. Ann Emerg Med 2016;67:316-17.

27 Gosbee J, Anderson T. Human factors engineering design demonstrations can enlighten your RCA team. Qual Saf Health Care 2003;12:119-21.
28 Varkey P, Karlapudi SP, Bennet KE. Teaching quality improvement: a collaboration project between medicine and engineering. Am J Med Qual 2008;23:296-301.

29 Xiao Y, Fairbanks RJ. Speaking systems engineering: bilingualism in health care delivery organizations. Mayo Clin Proc 2011;86:719-20.

30 NYPORTS-The New York Patient Occurrence and Tracking System—Annual Report 1999. https://www.health.ny.gov/press/ releases/2001/nyports/nyports.htm. 2001 (accessed 8 Sep 2015).

31 NYPORTS-SECTION 2: CLINICAL DEFINITIONS MANUAL. http://www.nashp.org/sites/default/files/NY_ PORTS_Clinical_Definitions.pdf. Published 2005 (accessed 8 Sep 2015).

32 NCC MERP Taxonomy of Medication Errors. Rockville, MD, 2007. https://www.nccmerp.org/sites/default/files/ taxonomy2001-07-31.pdf

33 Greenhalgh T, Taylor R. Papers that go beyond numbers (qualitative research). $\mathrm{Br}$ Med J 1997;315:740-3.

34 Pope C, Ziebland S, Mays N. Qualitative research in health care. Analysing qualitative data. Br Med J 2000;320:114-16.

35 Green M. Safety Hierarchy: Design Vs. Warnings. http://www. visualexpert.com/Resources/safetyhierarchy.html (accessed 8 Sep 2015).

36 Noble DJ, Pronovost PJ. Underreporting of patient safety incidents reduces health care's ability to quantify and accurately measure harm reduction. J Patient Saf 2010;6:247-50.

37 Rothschild JM, Landrigan CP, Cronin JW, et al. The Critical Care Safety Study: the incidence and nature of adverse events and serious medical errors in intensive care. Crit Care Med 2005;33:1694-700. http://www.ncbi.nlm.nih.gov/entrez/query. fcgi? cmd = Retrieve\&db=PubMed\&dopt $=$ Citation\&list_ uids $=16096443$

38 Rasmussen J. The role of error in organizing behavior. Ergonomics 1990;33:1185-99.

39 Joint Comission. Framework for Conducting a Root Cause Analysis and Action Plan. https://www.jointcommission.org/ framework_for_conducting_a_root_cause_analysis_and_action plan/. Published 2013 (accessed 8 Aug 2016). 